\title{
Activity Affects Dendritic Shape and Synapse Elimination during Steroid Controlled Dendritic Retraction in Manduca sexta
}

\author{
Carsten Duch and Tim Mentel \\ Institute of Biology and Neurobiology, Free University Berlin, 14195 Berlin, Germany
}

\begin{abstract}
Insect metamorphosis is a compelling example for dendritic and synaptic remodeling as larval and adult behaviors place distinct demands on the CNS. During the metamorphosis of the moth, Manduca sexta, many larval motoneurons are remodeled to serve a new function in the adult. During late larval life, steroid hormones trigger axonal and dendritic regression as well as larval synapse elimination. These regressive events are accompanied by stereotypical changes in motor behavior during the so-called wandering stages. Both normally occurring changes in dendritic shape and in motor output have previously been analyzed quantitatively for the individually identified motoneuron MN5. This study tested whether activity affected steroid-induced dendritic regression and synapse disassembly in MN5 by means of chronically implanted extracellular electrodes. Stimulating MN5 in vivo in intact, normally developing animals during a developmental period when it usually shows no activity significantly slowed the regression of high-order dendrites. Both physiological and anatomical analysis demonstrated that reduced dendritic regression was accompanied by a significant reduction in larval synapse disassembly. Therefore, steroid-induced alterations of dendritic shape and synaptic connectivity are modified by activity-dependent mechanisms. This interaction might be a common mechanism for rapid adjustments of rigid, inflexible, hormonal programs.
\end{abstract}

Key words: ecdysis; activity-dependent development; insect; neuromuscular system; motor terminal; hormone

\section{Introduction}

The postembryonic acquisition of behavior requires alterations in neuronal circuitry, which comprise specific changes in neuronal structure, membrane properties, and synaptic connectivity. Insect metamorphosis is a compelling example for dendritic and synaptic remodeling as larval and adult behaviors place distinct demands on the CNS (Consoulas et al., 2000; Tissot and Stocker, 2000). Because metamorphosis is controlled by ecdysteroids [including 20-hydroxyecdysone (20HE)], holometabolous insects have proven useful model systems to analyze steroid effects on dendritic shape (Weeks and Truman, 1986; Truman, 1990; Levine et al., 1995, 1996; Levine and Weeks, 1996). Steroids also affect dendritic outgrowth in hypothalamic slice cultures (ToranAllerand et al., 1999), cyclical alterations in dendritic spine density on rat hippocampal pyramidal cells during the estrous cycle (Woolley, 1998), and the maturation of the song-control system in birds (Bottjer and Arnold, 1997). Because dendritic shape is affected by activity in many systems (McAllister, 2000; Cline, 2001; Wong and Gosh, 2002; Libersat and Duch, 2004), a possible

Received April 21, 2004; revised Sept. 13, 2004; accepted Sept. 14, 2004.

We gratefully acknowledge the support by the Deutsche Forschungsgemeinschaft to C.D. (DU 331/2-3; SFB 515 , A7). We thank Dr. R. B. Levine (ARL Division of Neurobiology, University of Arizona, Tucson, AZ) for many helpfu comments on this manuscript. We thank J. F. Evers (Institute of Biology and Neurobiology, Free University Berlin, Berlin, Germany) for his expert help with the reconstruction tools for quantitative evaluation of neuronal shape and labeled protein distribution.

Correspondence should be addressed to Carsten Duch, Institute of Biology and Neurobiology, Free University Berlin, Koenigin-Luise Str. 28-30, 14195 Berlin, Germany. E-mail: duch@neurobiologie.fu-berlin.de.

DOI:10.1523/JNEUROSCI.3189-04.2004

Copyright $\odot 2004$ Society for Neuroscience $\quad$ 0270-6474/04/249826-12\$15.00/0 functional interplay between hormonal cues and neural activity seems logical, but only few studies have addressed this interaction (Woolley and McEwen, 1994; Woolley et al., 1997). An attractive hypothesis is that activity might serve as a modulator of rigid, nonflexible hormone actions.

During the metamorphosis of the moth, Manduca sexta, many larval motoneurons are remodeled to serve a new function in the adult (Consoulas et al., 2000). A surge in the 20HE titer during the prepupal peak (see Fig. 1C) triggers dendritic (see Fig. 1A) and axonal regression as demonstrated by hormonal manipulation experiments (see Fig. 1B) (Weeks and Truman, 1985, 1986; Levine and Weeks, 1989; Fahrbach and Weeks, 2002; Knittel and Kent, 2002). During the loss of larval behaviors, dendritic regression is accompanied by larval synapse elimination (Streichert and Weeks, 1995; Gray and Weeks, 2003). During pupal life, the formation of adult dendrites serves the integration into newly formed networks (Duch and Levine, 2000). Such changes in dendritic shape have been analyzed quantitatively throughout metamorphosis in the individually identifiable flight motoneuron, MN5 (Libersat and Duch, 2002).

During late larval life, structural changes of MN5 are accompanied by a defined sequence of motor behaviors. Five days before pupal ecdysis, wandering starts with the cessation of feeding (day W0). This is followed by crawling and burrowing at W1 and W2 and a phase of reduced movement that leads into a quiescent period underground at W3 and W4 (Reinecke et al., 1980; Dominick and Truman, 1984a,b). Pupal ecdysis occurs at W4 (Reinecke et al., 1980; Truman et al., 1980; Miles and Weeks, 1991). 
By means of chronically implanted extracellular electrodes at selected nerves in intact, freely moving, and normally developing animals, we demonstrated recently that the activity of MN5 is regulated in a stereotypical manner during the wandering stages (see Fig. $1 C, D$ ), and that stage-specific activity patterns induce axonal outgrowth (Duch and Mentel, 2003).

This study tested whether dendritic shape was also affected by neural activity. The results showed that steroid-induced dendritic regression and larval synapse elimination were modulated by increased neuronal activity.

\section{Materials and Methods}

Animals. Manduca sexta (L) obtained from a laboratory culture were reared on artificial diet (Bell and Joachim, 1976) under a long-day photoperiod regime ( $17 / 7 \mathrm{hr}$ light/dark cycle) at $\sim 26^{\circ} \mathrm{C}$. Both chronological and morphological criteria were used for staging of animals (Nijhout and Williams, 1974; Bell and Joachim, 1976; Dominick and Truman, 1984a). For the prepupal stages, W0 signifies the first day of wandering, and W1-W4 the remaining larval days (see Fig. 1C). Pupal ecdysis occurs at day W4. Dendritic regression of MN5 starts at W2 and is continuous until the third day of pupal life, stage P2 (Duch and Levine, 2000; Libersat and Duch, 2002). Chronic extracellular recordings can be conducted throughout the life of the fifth larval instar (V) until the first day of pupal life (P0), because the wires are lost during the pupal molt (see below). Therefore, this study examined animals during the last $3 \mathrm{~d}$ of larval life, W2 to P0. Stage W3 was defined by the eversion of the head revealing a characteristic triangle on the posterior edge of the head. Stage W3 lasted $\sim 24 \mathrm{hr}$ and was subdivided by chronological criteria into three substages [W3 early (W3e), W3 mid (W3), and W3 late (W31)] of equal duration. Depending on how long the development from stage W3 to W4 lasted, these substages were adjusted into three subsequent periods, each of which lasted between 6 and $10 \mathrm{hr}$. W3 never lasted $<18 \mathrm{hr}$, and animals that remained in stage W3 for longer than $30 \mathrm{hr}$ were regarded to be developmentally delayed and discarded. Stage W4 was defined by the appearance of the brown bar on the dorsal metathoracic segment and lasted $\sim 24 \mathrm{hr}$ (Truman et al., 1980). Stage W4 was subdivided into stage W4 early (W4e), W4 mid (W4), and W4 late (W41). W4e and W4 were defined as subsequent substages of equal duration covering the period between the appearance of the brown bar and the first sign of cuticular shrinkage on the first abdominal segment [anterior shrinkage (Truman et al., 1980); corresponding to W4a (Consoulas et al., 1996)] and lasted $\sim 8 \mathrm{hr}$ each. W4l was the remaining time until pupal ecdysis occurred, lasted $4-8 \mathrm{hr}$, and corresponded to stage $\mathrm{W} 4 \mathrm{~b}$ as published by Consoulas et al. (1996). Motor activity was recorded continuously from animals that developed from W2e to W31 or W4e. Then, chronic extracellular stimulations were conducted during stage W4 or W4l. Animals were put on ice for further dissection $5 \mathrm{hr}$ after the onset pupal ecdysis.

Chronic extracellular recordings. Bipolar hook electrodes for chronic implantations were manufactured from steel wires of $25 \mu \mathrm{m}$ diameter insulated to the tip. Two wires were aligned parallel to each other and fixed on a coverslip by complete coverage with Kwik-Cast/Kwik-Sil two component silicon glue (World Precision Instruments, Sarasota, FL). Subsequently, the silicon and the insulation at the very tips were removed only on the upper side of the wires, and the half-blank tips were bent to form fine hooks of $\sim 100 \mu \mathrm{m}$ length, with the blank part on the inside. The silicon cover of the electrode wires was $3 \mathrm{~cm}$ long, and the wires were left an additional $30 \mathrm{~cm}$ long to connect them to an amplifier (model 1700; A-M Systems, Carlsborg, WA). Extracellular signals were differentially amplified 1000-fold, digitized with an analog-to-digital converter (Digidata 1320A; Axon Instruments, Foster City, CA) and recorded continuously for several days at a sampling frequency of $10 \mathrm{kHz}$ with pClamp8 software (Axon Instruments) on a personal computer. The continuous data trace was broken into subsequent periods of $1 \mathrm{hr}$ to minimize file size $(137 \mathrm{MB} / \mathrm{hr})$.

Electrode implantations. Animals were anesthetized by chilling on ice for $3 \mathrm{hr}$. The following electrode implantations were conducted in the cold room at $4^{\circ} \mathrm{C}$ to minimize bleeding and to prevent movement. Animals were placed ventral side up in a large sylgard dish. The four anterior- most legs were held with lassos made from stainless steel wire, and the animal was slightly stretched to visualize mesothoracic nerve 1 from the outside. A large trachea served as landmark to record the same site of nerve 1 in different animals (Duch and Mentel, 2003). A fine cut of $\sim 200-300 \mu \mathrm{m}$ length was made just above the nerve into the larval cuticle. Then, the nerve was lifted with a glass hook, and the electrode was placed under the nerve with a micromanipulator. Subsequently, the electrode was slightly lifted to remove surrounding hemolymph. The nerve was fixed to the hooks with a 1:3 mixture of Kwik-Cast/Kwik-Sil that hardened within $20 \mathrm{sec}$ to completely insulate the hooks and the nerve. Then, the electrode was pushed back through the incision into the thorax, the wound was closed with tissue glue, and the silicon part of the electrode was glued to the lateral side of the animal toward the abdomen. Implanted animals were placed into cylindrical wooden chambers of 10 $\mathrm{cm}$ length and $2 \mathrm{~cm}$ diameter as in normal laboratory culture conditions. These were placed into a faraday cage to decrease electrical noise. Recordings were stable throughout the $3 \mathrm{~d}$ between stage W2 and larval-topupal ecdysis. Animals attempted complete ecdysis but were unable to shed the larval cuticle at the insertion site of the silicon electrode. Therefore, recordings were terminated after $2 \mathrm{hr}$ of ecdysis behavior. Staging of animals by external criteria was conducted every $8 \mathrm{hr}$.

At the recording site, nerve 1 contained the axons of the motoneurons MN1-MN5, the larger diameter axon of a motoneuron with its soma located in the midline of the prothoracic ganglion and the axons of six smaller motoneurons, two of which were located in the prothoracic ganglion and four of which were located in the mesothoracic ganglion, and the very thin axons of an octopaminergic ventral unpaired median neuron located in the mesothoracic ganglion (Duch and Mentel, 2003).

Chronic stimulation of motoneurons. To determine the effects of spiking activity, stimulation of nerve 1 was conducted with an A-M Systems stimulus generator (model 2100). Individual pulses were set to $0.1 \mathrm{msec}$ duration and $8 \mathrm{~V}$ amplitude for antidromic in vivo stimulation of the motoneurons MN1-MN5 during normal development. These pulse parameters induced no current spread and were also used in previous studies for antidromic identification of the motoneurons MN1-MN5 in the isolated ganglion preparation. By simultaneous intracellular recording in animals with chronically implanted extracellular electrodes, we have shown previously (Duch and Mentel, 2003) that each extracellularly applied stimulus resulted in one spike in MN5. Therefore, the stimulation protocol resembled the induced spiking activity in MN5. Stimulation amplitude and duration were just above threshold for inducing spikes in the large-diameter motoneuron axons, which makes it unlikely that other axons were also stimulated (Duch and Levine, 2000, 2002). To test the role of motoneuron activity for their structural remodeling nerve 1 was stimulated for $12 \mathrm{hr}$ with bursts of $1 \mathrm{sec}$ duration, $10 \mathrm{~Hz}$ frequency, and an interburst period of $10 \mathrm{sec}$. Individual pulses within each burst were of 0.1 msec duration and $8 \mathrm{~V}$ amplitude.

Intracellular identification of MN5. In all preparations, chronic recordings and stimulations were followed by intracellular recordings and staining of MN5. Animals were dissected carefully, dorsal side up, to prevent destruction of the nerve with the electrode implant. The mesothoracic ganglion was placed on a wax-coated platform, a suction electrode was placed on nerve 1 far more proximal than the site of the chronic electrode, and MN5 was impaled as described previously (Duch and Levine, 2000).

Intracellular recording and staining and synaptotagmin immunocytochemistry. A glass microelectrode (20-25 $\mathrm{M} \Omega$ with dye solution) was filled with $7 \%$ neurobiotin in $2 \mathrm{~m}$ potassium acetate to fill the MN5 intracellularly by injection of $3 \mathrm{nA}$ depolarizing current for $30 \mathrm{~min}$. Before dye fills impaled motoneurons, MN5s were recorded in situ in bridge mode (Axoclamp 2B) from their somata to measure resting membrane potential, input resistance, and spontaneously occurring postsynaptic potentials (PSPs) resulting from spontaneous spiking of unidentified neurons presynaptic to MN5. The ganglia were fixed with $7^{\circ} \mathrm{C}$ cold $4 \%$ paraformaldehyde for $2 \mathrm{hr}$ and rinsed for $1 \mathrm{hr}$ in PBS. Then, dehydration in an ascending ethanol series (50, 70, 90, 100\%; 15 min each) was followed by lipid extraction in three steps. First, an ethanol/methylsalicylate mixture (1:1) was applied for $10 \mathrm{~min}$. This was followed by $10 \mathrm{~min}$ methylsalicylate and $10 \mathrm{~min}$ methylsalicylate/xylene (1:1) to increase an- 
tibody permeability and reduce background staining. Subsequently, these steps were conducted in reverse order. After rehydration in a descending ethanol series, ganglia were bathed in $0.5 \%$ Triton X-100 PBS for $2 \mathrm{hr}$. If immunolabeling was performed, the ganglia were preincubated in 10\% normal goat serum, $0.5 \%$ Triton X-100 PBS ( $0.1 \mathrm{M})$ for $1 \mathrm{hr}$, incubated in primary antibody (rabbit anti-synaptotagmin) for $2 \mathrm{~d}$, and washed in PBS for $1 \mathrm{hr}$. The synaptotagmin antibody was a gift from Dr. L. P. Tolbert (ARL Division of Neurobiology, University of Arizona, Tucson, AZ). It had been raised against the unique $\mathrm{N}$ terminus of Manduca sexta synaptotagmin and has been shown to specifically detect Manduca synaptotagmin (Dubuque et al., 2001). The neurobiotin staining was visualized by coupling to cyanine 3 (Сy3)-streptavidin (1:750 streptavidin-Cy3 in $0.05 \%$ Triton X-100 PBS). In case of previous treatment with primary antibodies, Cy5-coupled mouse anti-rabbit (Jackson ImmunoResearch, West Grove, PA) secondary antibody was coapplied in 1:200 concentration. After washing in PBS for $1 \mathrm{hr}$, the preparation was dehydrated, thereafter cleared in a mixture of ethanol and methylsalicylate (1:1) for $10 \mathrm{~min}$, and mounted in 100\% methylsalicylate. Acquisition of confocal image stacks was done immediately.

Confocal microscopy. Digital images were captured on a Leica (Bensheim, Germany) TCS SP2 scanning confocal microscope. All scans were conducted with a Leica HCX PL APO CS $40 \times$ oil immersion objective (numerical aperture, 1.25). Optimal physical resolution with the $40 \times$ oil lens used is $0.1 \times 0.1 \times 0.28 \mu \mathrm{m}$. At $3.5 \times$ zoom and a $\mathrm{z}$-step increment of $0.3 \mu \mathrm{m}$, the resulting voxel resolution was $0.1 \times 0.1 \times 0.3 \mu \mathrm{m}$, thus matching the optimal physical resolution. However, optical resolution is also limited to approximately half excitation wavelength but may further be degraded to some degree by inhomogeneous tissue properties. All images were obtained in simultaneous acquisition mode (i.e., the emitted fluorescence light of both synchronously excited dyes was divided by an acoustic-optical beam splitter to be detected by two separate photomultipliers). Thus, no image misalignment occurred as a result of an error in scan mirror positioning. Images were further processed using Amira (TGS, San Diego, CA) and Corel Draw 8 software (Corel, Ottawa, Ontario, Canada). All original images shown are the projections of all optical planes of a given stack into one focal plane to visualize all dendrites of MN5 in one two-dimensional image.

Correction for chromatic aberration. To correct for chromatic aberration, a neuron was filled with biotin and double labeled with Cy3- and Cy5-coupled streptavidin (Jackson ImmunoResearch). An image stack was obtained as described above. The misalignment between both structures was measured and applied onto later acquired image stacks of equally treated specimens (Wouterlood et al., 1998).

Three-dimensional reconstructions of dendritic structure and morphometric analysis. To obtain quantitative data on dendritic morphology, the arborizations of MN5 were reconstructed from confocal image stacks. Confocal image stacks were acquired as described above and analyzed with Amira software. A semiautomatic tool to create three-dimensional reconstructions in the Amira environment with higher accuracy compared with conventional automatic or manual method was recently developed and described in detail by Schmitt et al. (2004). Briefly, a semiautomatic reconstruction procedure is realized by adapting the snake algorithm (Kass et al., 1988) to trace approximately tubular neuronal structures in confocal image stacks. User interaction is needed to define branch point hierarchy, whereby the algorithm automatically determines the midlines and diameters of interconnecting link segments at a userpredefined step size. This step size was set to $0.5 \mu \mathrm{m}$ in all reconstructions conducted. The resulting tubular reconstruction of the neuronal tree contains precise information on length, diameter, orientation, and branching pattern. To access exact surface and volumetric measures, an adapted geodesic active contour algorithm (Caselles et al., 1997) is used to fit the idealized tubular reconstruction to actual image data, resulting in precise boundary definition.

Analysis of labeled presynaptic protein distribution along motoneuronal dendritic surfaces. The exact surface definitions obtained by fitting the tubular reconstructions to the actual image data with an adapted geodesic contour algorithm allow analysis of labeled molecule distribution along neuronal surfaces in submicron resolution (Schmitt et al., 2004). Briefly, all anti-synaptotagmin label image intensities more distant than
$0.3 \mu \mathrm{m}$ from the dendritic surface were set to zero. The remaining synaptotagmin label was located within $0.3 \mu \mathrm{m}$ from the dendritic surface of MN5 and was used to calculate the mean staining density around each patch of the triangulated surface reconstruction. The calculated synaptotagmin staining intensities can be visualized as a color code by projecting each patch of the surface reconstruction onto the individual cylindrical compartments. This enables analysis of geometrical relationship between staining distribution and the morphology of the neuron. Warmer colors represent highest synaptotagmin staining intensity localized in the terminals of unidentified presynaptic neurons, which are located $0.3 \mu \mathrm{m}$ or closer to the dendritic surface of the motoneuron (see Fig. 5). Because synaptic protein markers like synapsin 1 and synaptotagmin can be used to mark the sites of synapses in the insect CNS, and electron micrographs have shown that presynaptic vesicles are found in distances up to $0.2-0.3 \mu \mathrm{m}$ from the postsynaptic membrane (Peters et al., 1985; Watson and Schurmann, 2002), this offers a method to assess putative synapse distribution throughout entire dendritic trees on the light microscopy level (Schmitt et al., 2004) (J. F. Evers and C. Duch, unpublished data).

Statistics. For paired statistical comparison of two independent groups, one-sided paired Student's $t$ tests were conducted. All morphometric parameters obtained from pairs of stimulated and nonstimulated motoneurons, with each pair derived from the same animal were tested in this way. Physiological parameters like the amplitude and the frequency of postsynaptic potentials derived from two different experimental groups (stimulated and nonstimulated animals) were tested by onesided unpaired Student's $t$ tests. Differences were judged statistically significant at $p \leq 0.01$. $t$ tests were conducted with Microsoft (Seattle, WA) Excel. To test for statistically significant differences within more than two experimental groups, one-way ANOVA was conducted (significance level, $p \leq 0.01$ ). If ANOVA revealed significant differences within the data, the individual experimental groups were compared by post hoc comparisons between means by the Newman-Keuls test (Zar, 1984) (significance level, $p \leq 0.05$ ). ANOVA and post hoc Newman-Keuls tests were conducted with Statistica (StatSoft, Tusla, OK).

Solutions. Saline consisted of the following (in mM): $140 \mathrm{NaCl}, 5 \mathrm{KCl}, 4$ $\mathrm{CaCl}_{2}, 28$ D-glucose, 5 HEPES, $\mathrm{pH}$ was adjusted to 7.4 using $1 \mathrm{~m} \mathrm{NaOH}$.

\section{Results \\ Stimulation of MN5 during the normal decline of activity affects dendritic shape}

During the wandering stages $\mathrm{W} 3$ and $\mathrm{W} 4$, a marked decline of motor activity in vivo coincides in time with a number of structural modifications that are important for the normal development of the adult neuromuscular system (Fig. 1C) (Duch and Mentel, 2003). During this time, the motoneuron MN5 undergoes drastic regression of its dendritic (Fig. 1A) (Duch and Levine, 2000; Libersat and Duch, 2002) and axonal arborizations (Fig. 1B) (Duch et al., 2000). Shortly after pupal ecdysis and, thus, after the motoneurons have participated in ecdysis behavior, motor terminals start to grow onto the developing adult muscle anlagen (Duch et al., 2000), and within the CNS, the formation of prominent dendritic growth cones precedes the sprouting and growth of new adult dendrites (Duch and Levine, 2000; Libersat and Duch, 2002). The normal decline in firing activity is important for the retraction of the axon terminals of MN5, and ecdysis-like activity patterns are sufficient to induce axonal outgrowth (Duch and Mentel, 2003). To test whether activity may also affect steroid-induced dendritic regression and larval central synapse elimination, MN5 was stimulated in intact normally developing animals between the stages $\mathrm{W} 3$ and $\mathrm{W} 4$. A total of 15 such stimulation experiments were conducted. In all animals, chronic recording electrodes were implanted at stage W2l or W3e. Recordings were maintained in unrestrained animals until the typical cessation of motor activity between stages W3l and W4 occurred (Fig. 1D) (Duch and Mentel, 2003). External staging 


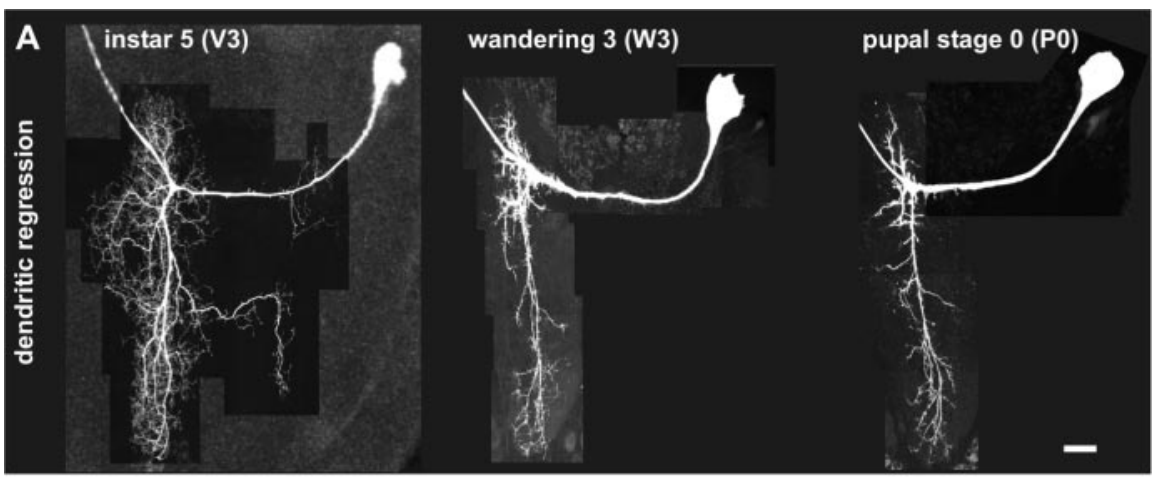

B

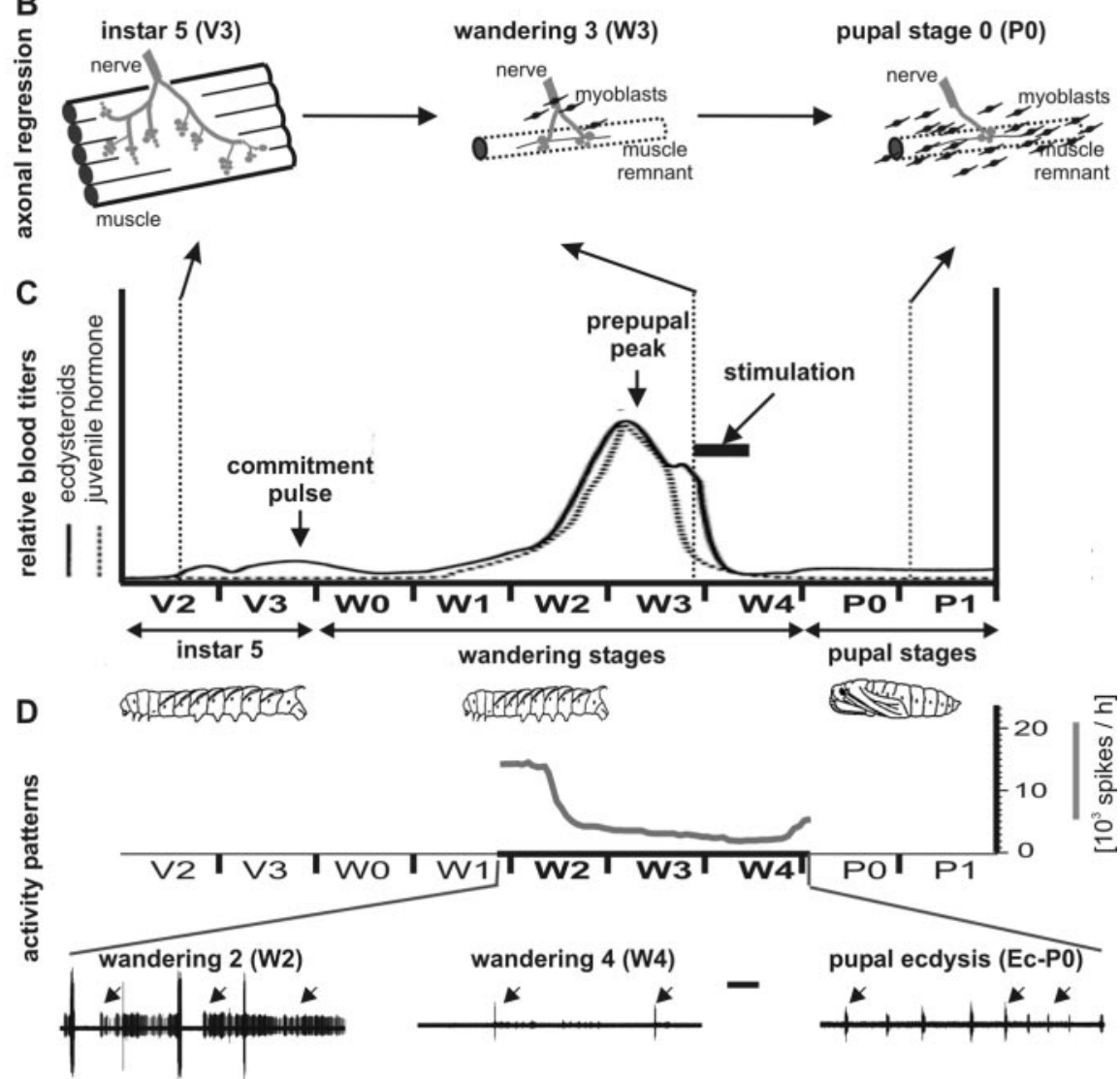

Figure 1. Regression of MN5 dendrites and axons, developmental changes in hormone titers, and stereotypical changes in MN5 spiking activity during the larval-pupal transformation of Manduca. A, Projection views from confocal image stacks of MN5 at larval day V3 (left), the third day of wandering (W3; middle), and the first day of pupal life (P0; right) showing MN5 dendritic retraction. $B$, Schematic summary of motoneuron-muscle remodeling during the developmental time relevant to this study. Larval (V) muscle fiber innervation retracts during wandering stages $(W)$, degenerating muscles, myoblasts accumulate. At the first day of pupal life ( $\mathrm{PO})$, the adult muscle anlagen is formed by the fusion of myoblasts with the remnants of larval muscle fibers. During later pupal life, new motor terminals grow over the developing muscle (Duch et al., 2000). C, The timeline relevant to this study illustrates changes in relative hemolymph levels of ecdysteroids (solid line) and juvenile hormone (dashed line) from second day of the fifth larval instar (V2) to second day of pupal life [hormone titers redrawn from Bollenbacher et al. (1981) and Riddiford and Truman (1994)]. A small rise in ecdysteroids (20E) occurs on day V3, the commitment pulse, and a larger rise in $20 \mathrm{E}$ spans days W1 to $\mathrm{P0}$, the prepupal peak. The rise of the prepupal peak of 20E triggers dendritic regression in motoneurons (Weeks and Truman, 1985, 1986; Weeks et al., 1992). V2, V3, Day 2, 3 of the fifth larval instar; W0 to W4, days of the wandering fifth instar; P0, day of ecdysis to the pupal stage; P1, next day of pupal life; bold black arrow signifies the time period of in vivo mesothoracic nerve 1 stimulation. D, Summary of the activity of motoneurons with their axon in the mesothoracic nerve 1, as recorded with chronically implanted electrodes in vivo in normally developing animals (Duch and Mentel, 2003). The graph relates to the same timescale as the hormone titers in Cand shows activity of the MN1-5 as recorded in vivo. Representative bouts of activity as recorded from nerve 1 in vivo are shown for selected developmental stages. Motor spike amplitudes of MN1-5 are marked by arrows. Scale bars: $A, 50$ $\mu \mathrm{m} ; D, 20 \mathrm{sec}$ criteria were used to confirm that the animals had developed to stage W3l or W4e. Then, nerve 1 was stimulated for $12 \mathrm{hr}$ with bursts of $1 \mathrm{sec}$ duration, $10 \mathrm{~Hz}$ frequency, and an interburst period of $10 \mathrm{sec}$ Stimulation was terminated at stage W4l (Fig. 1C). All animals attempted pupal ecdysis and were put on ice for intracellular staining of MN5 $5 \mathrm{hr}$ after the onset of ecdysis motor patterns (Fig. $1 D$ ). In 12 experiments, the stimulated MN5 was recorded and stained intracellularly at stage P0. In three experiments, MN5 was stained on both sides of the ganglion at stage P0. Because nerve 1 was stimulated on one side of the animal only, the contralateral side served as an internal control having the same hormonal state as the experimental side. In addition, the comparison of MN5 on the stimulated and the control side of the same animal ensured that putative structural differences were not attributable to developmental differences among animals.

Induced activity of MN5 at W31/W4e, when no natural activity was observed in vivo, clearly affected dendritic structure (Fig. 2). Although overall dendritic shape appeared rather similar in stimulated dendritic trees compared with control dendritic trees, many more fine branches were present in the stimulated versus the control trees (Fig. 2A). However, all 12 stimulated motoneurons clearly showed dendritic regression despite the stimulation experiment, because their dendrites contained fewer branches and showed a smaller total length compared with stage W3l or stage W4e dendritic trees. This was not the case in electrode implanted but nonstimulated control animals (data not shown). To visualize the dendritic tree from different perspectives and to quantify the effects of activity on dendritic branching, geometric reconstructions were conducted. Representative examples for three-dimensional reconstructions of the dendritic trees of MN5 from two animals on the stimulated side and on the contralateral internal control side are depicted in Figure $2 B$ (i, animal 1; ii, animal 2). For better visualization, each dendritic tree is shown as a xy-view and xz-view (Fig. 2B). The basic dendritic tree structure was similar on the control and stimulated side, but the stimulated trees contained more fine branches. This became particularly obvious in the xz-views. Geometric reconstructions of stimulated and control dendritic trees were used also for a quantitative analysis of the effects of activity on dendritic shape. In total, three control neurons at stage W4e (the stage when 
stimulation was started), three unstimulated control neurons from implanted animals at stage $\mathrm{P} 0$, and four stimulated neurons from stage P0 were reconstructed. In addition, the morphometric parameters from three stage $\mathrm{P} 0$ neurons from nonimplanted animals, which were reconstructed in a previous study (Libersat and Duch, 2002), were used for quantitative comparison among different groups. During normal development, the number of dendritic branches and the total dendritic length (TDL) decreased significantly between stages W4 and P0 (Fig. $3 A, B$ ) (oneway ANOVA; post hoc Newman-Keuls test; $p<0.01)$. This decrease in dendritic complexity was reduced but not stopped by the stimulation of MN5, because the number of branches and TDL were significantly smaller in stimulated P0 motoneurons than in stage W4 motoneurons (Fig. $3 A, B$ ) (one-way ANOVA; post hoc Newman-Keuls test; $p<0.01$ ). In fact, in stimulated P0 motoneurons (TDL, $7189 \pm$ $1276 \mu \mathrm{m}$ ), only $40 \%$ of the normal decrease in TDL between W4 (10,010 \pm $1459 \mu \mathrm{m})$ and P0 $(5474 \pm 744 \mu \mathrm{m})$ occurred. The same was the case for the number of dendritic branches (Fig. $3 A, B$ ). The decrease in the amount of dendritic regression in stimulated motoneurons was statistically significant, because both the number of branches and TDL were significantly larger in stimulated compared with control stage $\mathrm{P} 0$ motoneurons (Fig. 3A,B) (one-way ANOVA; post hoc Newman-Keuls test; $p<0.01)$. To exclude the possibility that the observed differences in morphometric parameters between the groups might be attributable to stage-specific differences in the histological procedures or to other artifacts, we also tested for differences in a parameter that usually stayed constant among the stages under investigation. One such parameter was the mean dendritic length (MDL; average length of all individual dendritic segments of a tree), which had been shown previously to remain constant between the onset of wandering and P0 (Libersat and Duch, 2002). MDL was similar in all three experimental groups: $\mathrm{W} 4(4.7 \pm 0.1 \mu \mathrm{m}), \mathrm{P} 0$ control $(4.6 \pm 0.2 \mu \mathrm{m})$, and $\mathrm{P} 0$ stimulated (Fig. $3 C$ ) ( $4.6 \pm 0.3 \mu \mathrm{m}$; one-way ANOVA, $p=0.94)$. Thus, the observed differences in TDL and in the number of branches (Fig. $3 A, B)$ were not caused by artificial differences among the different groups.

An increased number of high-order branches and a larger TDL in stimulated dendritic trees compared with internal and stage-matched controls could in principle have two causes, either reduced dendritic regression or premature outgrowth. Several lines of evidence indicated that imposed activity slowed dendritic regression but most likely did not cause premature outgrowth. First, in stimulated animals, no obvious dendritic branches or subtrees were found, which were not present at earlier stages before stimulation. Dendritic tree shape in control stage W4 early animals (Fig. 3D) was very similar compared with stimulated
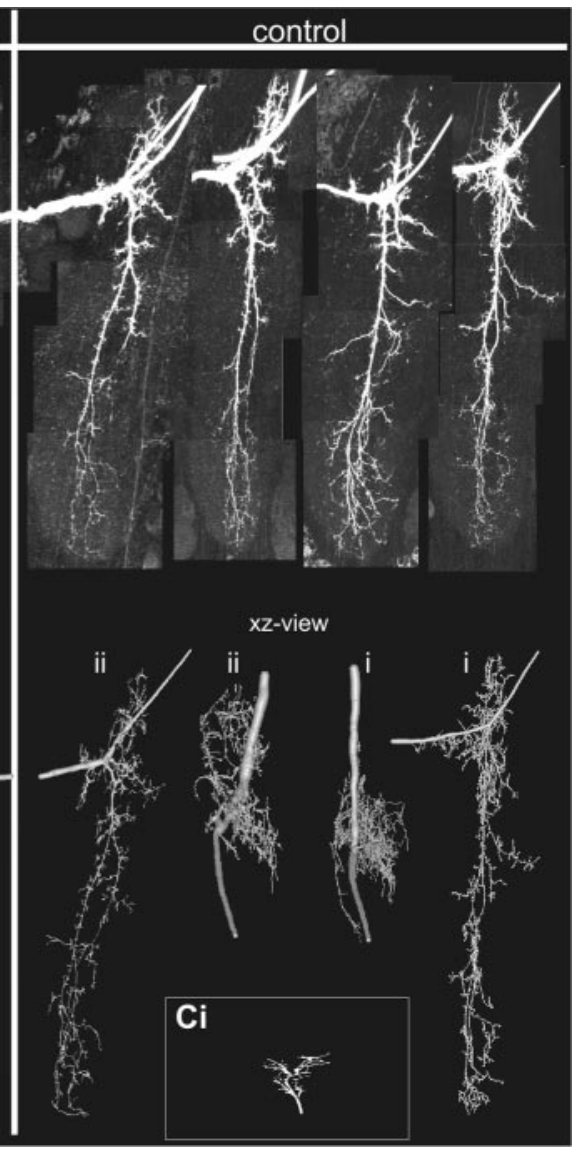

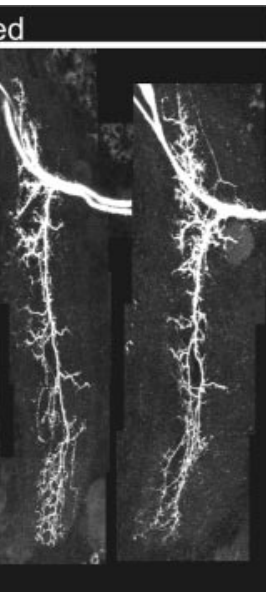

xz-view

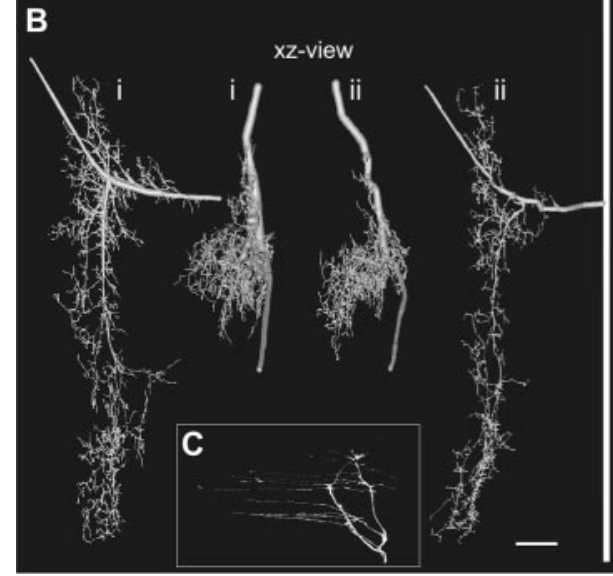

Figure 2. Dendritic shape in stimulated versus control MN5. A, Projection views from confocal image stacks of MN5 fixed and

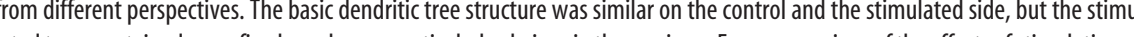
dendritic and axonal shape, the axonal projection patterns of the motoneurons MN1-MN5 are depicted from the stimulated $(C)$ and the control side (Ci) of one animal (modified from Duch and Mentel, 2003). Scale bar, $50 \mu \mathrm{m}$.

stage P0 dendritic trees (Fig. 2A). In contrast, dendritic trees from later control stages, when normal dendritic growth had occurred (Fig. 3E), showed many new dendritic branches that could easily be distinguished from those present at stage W4. The high constancy of dendritic shape in individually identified insect neurons like MN5 among animals (Libersat and Duch, 2002) allowed a good judgment on whether new branches were formed in the dendritic field. On this basis, we claim that stimulation induced reduced dendritic regression but no obvious new branch formation. Second, during normal development, dendritic outgrowth is preceded by the formation of prominent growth cones at the tips of all dendrites (Fig. 3E) (Duch and Levine, 2000; Libersat and Duch, 2002). These growth cones were not found after stimulating MN5 during late larval life. Therefore, imposed activity did not lead to the formation of dendritic growth cones, which were a prerequisite for outgrowth during normal development. Third, metric measures like TDL and the number of branches of stimulated MN5 at stage P0 laid between those of control developed stage W4 and stage P0 dendritic trees. This showed that regression had not stopped but strongly indicated that regression was slowed down. To further support this finding, branch-order analysis was conducted. This demonstrated that 

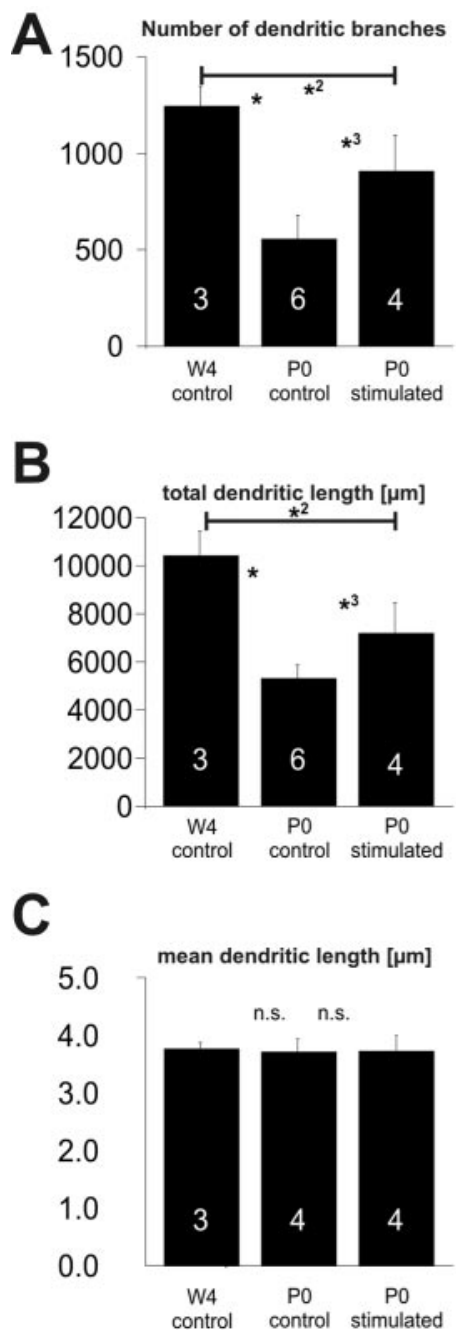
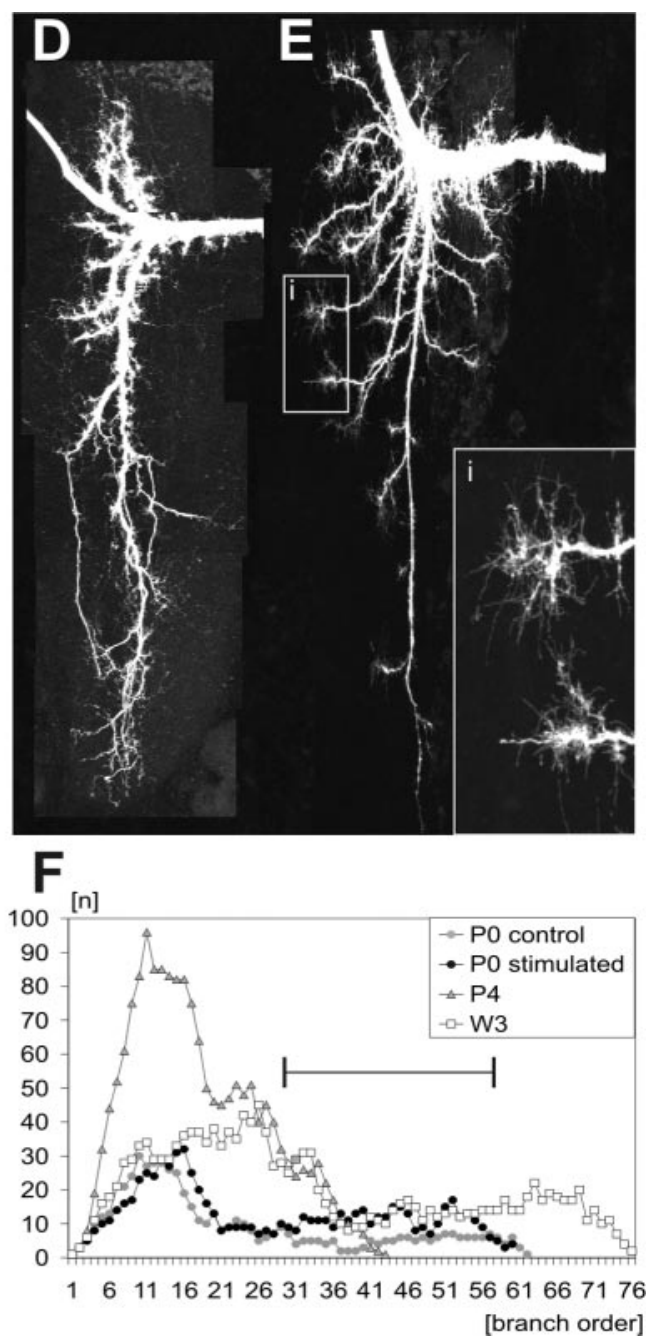

Figure 3. Quantification of dendritic shape in stimulated versus control MN5. $A-C$, The number of dendritic branches $(A)$, the total dendritic length (TDL; $B$ ), and the mean length of all dendritic segments (MDL; $C$ of MN5 were compared between control neurons at stage $\mathrm{W} 4$, control neurons at stage $\mathrm{P} 0$, and stimulated neurons at stage $\mathrm{P} 0$. The number of branches decreased significantly between stages W4 and P0 ( $A$, asterisk). This decrease was not stopped by the stimulation, because the number of branches was significantly smaller in stimulated P0 motoneurons than in stage W4 motoneurons ( $A$, asterisk 2$)$. Dendritic regression was decreased by stimulation, because stimulated $\mathrm{PO}$ motoneurons had significantly more branches than control P0 motoneurons ( $A$, asterisk 3). The significant decrease in TDL between stages W4 and P0 ( $B$, asterisk) was also not stopped but decreased by stimulation, because TDL was significantly smaller in stimulated P0 motoneurons than in stage W4 motoneurons ( $B$, asterisk 2) and significantly larger in stimulated PO motoneurons than control PO motoneurons ( $B$, asterisk 3). $C, M D L$ served as a control to account for putative differences in the histological procedure among the different groups, because it was previously shown to remain constant between W4 and P0 (Libersat and Duch, 2002). $n$ is indicated by the numbers in the bars. Asterisks indicate statistical significance (1-way ANOVA; post hoc Newman-Keuls test; $p<0.01$ ). D, Projection view of the dendritic tree of MN5 at an early stage W4. E, Projection view of the dendrites of MN5 at pupal stage P4 when dendritic growth started during normal development. Prominent growth cones were located at the tips of all dendrites (see inset $i$ ). $F$, Branch-order analysis for stages control W3 (open squares), control PO (gray circles), stimulated PO (black circles), and control P4 (gray triangles). The black bar signifies the branch orders for which stimulation increased the number of dendrites at stage P0. These branch orders were present at the earlier stage W3 but not during the first days of dendritic growth during normal development (stage P4).

control trees at W3 late contained many more high-order branches compared with $\mathrm{P} 0$ trees (orders 20-70) (Fig. 3F). Stimulated $\mathrm{P} 0$ trees also contained more high-order branches compared with control P0 trees (orders 30-60) (Fig. 3F), but in no order more branches than W3 late trees (Fig. $3 F$ ), indicating that no new branches were formed in any specific branch order. However, control stage P4 trees showed strongly increased branching in the lower orders up to 40 (Libersat and Duch, 2002). Therefore, during normal development, new branching occurred at lower orders, but stimulation led to the maintenance of the num- ber of high-order branches as occurring during earlier stages. Therefore, we concluded that stimulation induced reduced dendritic regression but not premature outgrowth. This also agreed with the finding that stimulation did not induce additional dendritic branches at earlier larval stages before dendritic regression started (data not shown). This was in contrast to previous findings on the effects of imposed activity on axonal structure, where stimulation induced significant new outgrowth (Duch and Mentel, 2003) (Fig. $2 C)$. Therefore, dendrites and axons of the same motoneurons were affected differently by increasing activity in stimulation experiments with chronically implanted electrodes.

\section{Stimulation of MN5 during the normal decline of activity affects larval synapse elimination}

It is plausible to assume that dendritic regression might be accompanied by synapse elimination. For Manduca accessory planta retractor motoneurons, it has been demonstrated recently that steroidinduced dendritic regression physically disconnects the motoneurons from the synaptic terminals of sensory neurons, producing synaptic weakening (Streichert and Weeks, 1995; Gray and Weeks, 2003). This agrees with our findings on dendritic regression and synapse elimination in motoneuron MN5 (Evers and C.D., unpublished data). However, if dendritic regression was functionally linked to synaptic weakening, stimulation might have causednot only decreased dendritic regression but also decreased synapse elimination. We tested this hypothesis physiologically and anatomically.

At stage P0, spontaneous PSPs resulting from the activity of unidentified neurons presynaptic to MN5 in the in situ preparation occurred at a higher frequency and showed a larger amplitude in stimulated compared with control motoneurons (Fig. 4). As an example, Figure $4 A$ shows traces of spontaneous activity recorded from a control and stimulated MN5 from the same ganglion. Although IPSPs occurred much more frequently than EPSPs (Fig. 4A) at all larval and early pupal stages, which were subjected to this study, both were included in the analysis. Stimulation did not change the ratio between spontaneously occurring IPSPs and EPSPs. Pooling the data from five stimulated and five control motoneurons clearly demonstrated that the frequency (control, 7.1 $\pm 4.2 \mathrm{~Hz}$; stimulated $14.0 \pm 6.9 \mathrm{~Hz}$ ) and the amplitude (control, $1.43 \pm 0.40 \mathrm{mV}$; stimulated, $1.92 \pm 0.39$ $\mathrm{mV}$ ) were significantly increased after in vivo stimulation (Fig. $4 B$ ) (Student's $t$ test; $p<0.01$ ). A quantitative comparison of PSP 

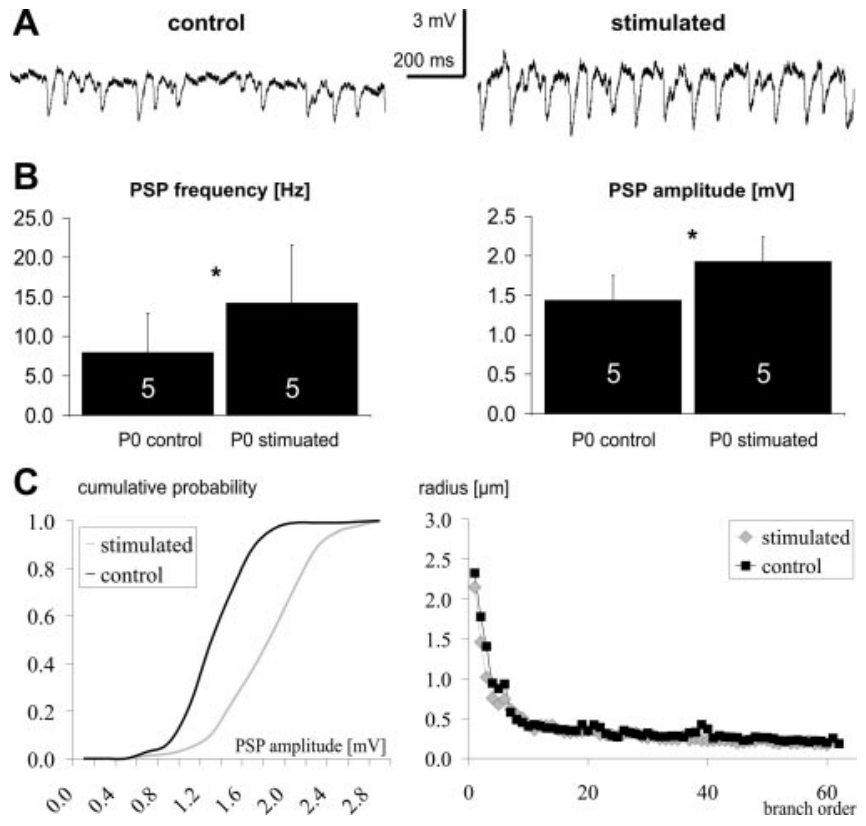

Figure 4. Quantification of PSP amplitude and frequency in stimulated versus control MN5. $A$, Recordings of spontaneously occurring PSPs in a control (left) and a stimulated (right) MN5 from the same animal. Both PSP amplitude and frequency were greater on the stimulated side. $B$, Quantitative comparison of PSP frequency (left) and PSP amplitude (right) averaged for five control and five stimulated MN5 seconds. Error bars represent SDs. Both parameters were significantly larger in the stimulated MN5 (Student's $t$ test; $p<0.01$ ). C, Cumulative probability plots of individual postsynaptic potentials (left) and dendritic diameters as a function of their branching order (right) for a control versus a stimulated MN5 from the same animal. Dendritic diameters were similar, but there was an increase in larger amplitude potentials in the stimulated MN5.

amplitude from a stimulated and contralateral control MN5 from the same animal further confirmed this result. Cumulative probability plots of individual postsynaptic potentials showed that there was an increase in larger amplitude potentials in the stimulated compared with the control MN5 from the same animal (Fig. 4C). Differences in PSP amplitude were not attributable to differences in input resistance $(58 \pm 2 \mathrm{M} \Omega)$, membrane potential $(-48 \pm 3 \mathrm{mV}$ ), or time constant, because these parameters were similar in the stimulated and control MN5 (Student's $t$ test; $p>0.2$ ). The same was the case for shape and amplitude of both the orthodromically and antidromically evoked action potential (data not shown). The larger PSP amplitude in MN5 on the stimulated side was also not caused by differences in passive conduction resulting from changes in dendritic architecture. Dendritic diameter is a crucial parameter for passive conduction of voltage signals. Plotting the diameters of all dendritic branches as a function of branch order for the stimulated and unstimulated control MN5 from the same animal showed that stimulation induced no changes in the diameters (Fig. 4C). Because the dendritic tree of the stimulated MN5 contained significantly more dendrites, shunting of PSP amplitude at branch points should have been stronger but not smaller compared with the control MN5. Therefore, the larger PSP amplitude in the stimulated motoneuron was most likely caused by more synaptic input compared with the control MN5. Both PSP amplitude and frequency of stimulated stage P0 motoneurons lay between those of control W4 (stage before stimulation) and control P0 animals. In contrast, in later stages when dendritic growth occurred during normal development, fewer and lower amplitude PSPs occurred compared with control P0 animals. This indicated that stimula- tion-induced decreases in dendritic regression were accompanied by a decrease in larval synapse disassembly.

We further tested whether there was an anatomical correlate to the physiological finding of reduced larval synapse elimination after in vivo stimulation. All spontaneously occurring PSPs were counted to compare synaptic input between stimulated and unstimulated neurons. The anatomical correlate would be the input synapse distribution throughout the entire dendritic tree. An exact analysis of the distribution of all input synapses throughout the dendritic tree could only be achieved by serial section Immunocytochemistry-electron microscopy studies, but this was not feasible for the dendritic trees of MN5 on both sides of the ganglia in unilaterally stimulated animals. However, in insects, the distribution of synapsin I-protein or synaptotagmin-protein is correlated with clustered synaptic vesicles at synaptic sites and is, therefore, useful in pinpointing synaptic loci in some neuropiles at the light microscopic level (Watson and Schurmann, 2003). We paired all intracellular stainings of MN5 with synaptotagmin immunocytochemistry in the same preparations. Synaptotagmin immunocytochemistry labeled the neuropile region around the dendritic field of MN5 (Fig. 5A). Moreover, single optical sections showed that individual synaptotagmin-immunopositive dots could easily be distinguished from each other (Fig. 5B). It has been shown previously that at the Manduca neuromuscular junction, the distribution of distinct synaptotagmin-immunopositive dots was almost identical to the distribution of activitydependent synaptic vesicle recycling sites in motor terminals (Consoulas and Levine, 1998). Therefore, it was likely that most synaptotagmin-positive dots (Fig. $5 A, B$ ) indicated putative presynaptic sites in the CNS. This is further supported by the finding that synaptotagmin spots in the neuropile show on average the same size that was reported for putative sensorimotor synapses described in Manduca abdominal ganglia at the same stages, based on contacts identified in confocal images (Gray and Weeks, 2003). Because the three-dimensional reconstructions used to analyze the structure of MN5 were conducted with a new method that provided very precise surface definitions (Schmitt et al., 2004), we could use the double stainings of MN5 and synaptotagmin profiles to estimate the distribution of putative input synapses throughout the entire dendritic tree of MN5 (see Materials and Methods) (Evers, S. Schmitt, M. Sibila, and Duch, unpublished data). The staining intensity of all synaptotagmin-positive dots within $0.3 \mu \mathrm{m}$ from the surface of the dendrites was used to calculate the mean staining density around each patch of the triangulated surface reconstruction. The resulting intensities were visualized as a color on the surface reconstruction. Warmer colors represented higher staining intensities of synaptotagminimmunopositive label located $0.3 \mu \mathrm{m}$ or closer to the dendritic surface (Fig. 5C). Visual inspection of the surface reconstruction within the stack of images for synaptotagmin label confirmed that sites of the dendritic surface signified by warm colors were, in fact, in close proximity to synaptotagmin-immunopositive profiles (Fig. 5C). This was further confirmed by visual inspection of single, unprocessed optical sections of double stainings of MN5 dendrites and synaptotagmin (Fig. $5 E-H$ ). At high scanning resolution, synaptotagmin spots could clearly be distinguished from each other, but only some were in close contact with the labeled dendrites (Fig. $5 F, H$ ). We found only very few synaptotagminimmunopositive dots inside the dendritic surface reconstruction, which is typical for efferent insect neurons (Watson and Schurmann, 2003). These were neglected in this type of analysis. Therefore, dendritic surface sites marked by warm colors were interpreted as sites of putative synaptic input. We do not claim that 

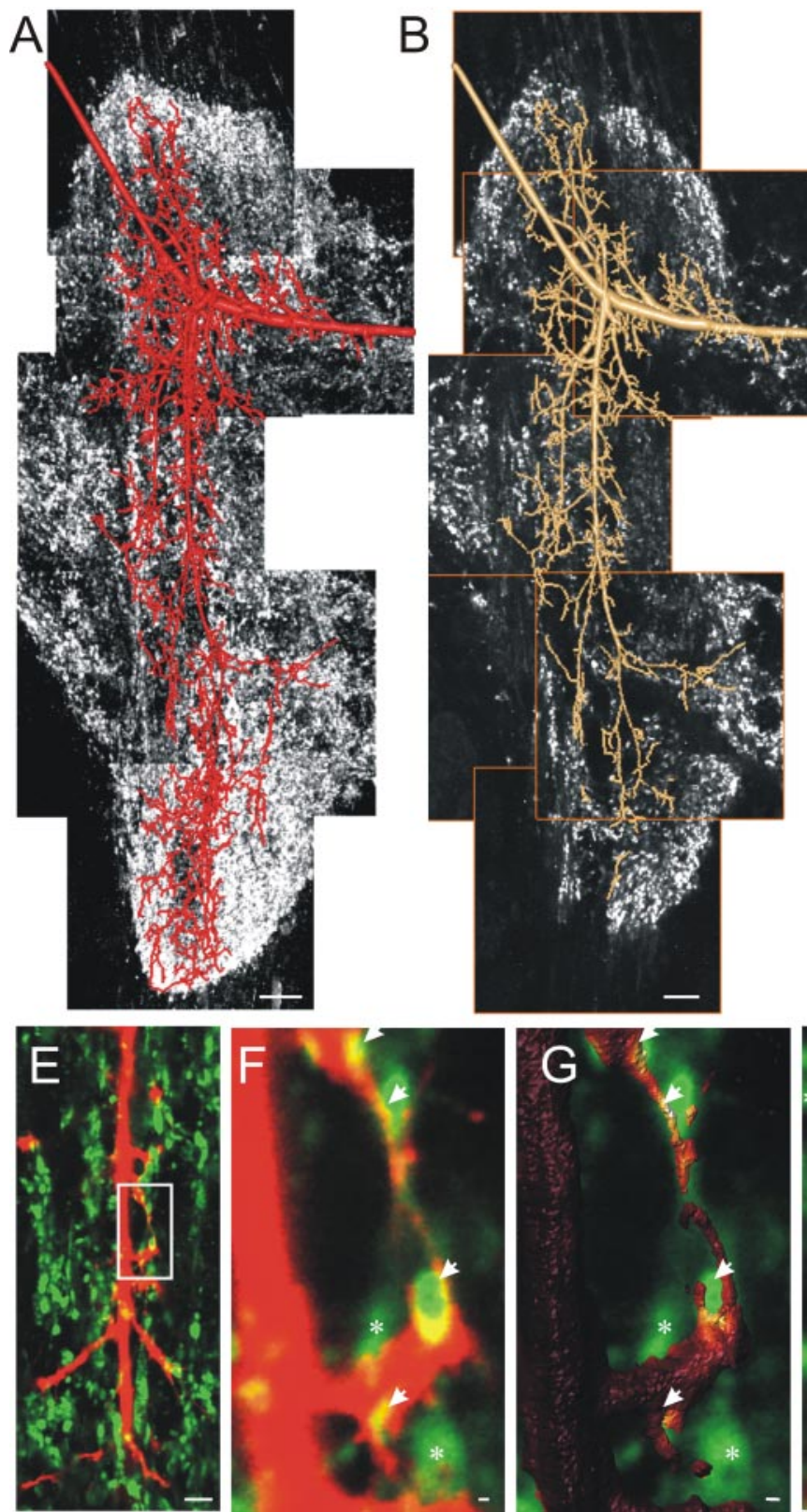

Figure 5. Localization of immunolabeled synaptotagmin profiles around the dendritic surface. $A$, Projection view of synaptotagmin immunocytochemistry around a geometric reconstruction of dendritic field of MN5 from a stimulated stage P0. Synaptotagmin label was found exclusively in neuropile regions. $B$, Single optical sections show that individual synaptotagmin-immunopositive dots could easily be distinguished from each other. The geometric reconstruction provides very precise surface definitions (Schmitt et al., 2004) and is used to estimate the distribution of synaptotagmin protein-containing putative input synapses throughout the entire dendritic tree of MN5 (see Materials and Methods). C, Selective enlargement of a single dendritic node in close association to two immunocytochemically stained synaptotagmin profiles. Synaptotagmin immunocytochemistry staining intensity within $0.3 \mu \mathrm{m}$ from the dendritic surface was used to calculate the mean staining density around each patch of the triangulated surface reconstruction. The resulting intensities were visualized with warmer colors representing higher staining intensities of synaptotagmin-immunopositive label. D, Application of the method shown in ( for the entire reconstruction shown in B.E, Unprocessed images of labeled dendrites (red) and nearby synaptotagmin-positive spots (green) in a single optical section. The white box indicates an area selectively enlarged in F. Arrowheads mark synaptotagmin spots (green), which are in contact with the labeled dendrite (red). The white asterisks mark synaptotagmin spots located further than $0.3 \mu \mathrm{m}$ from the labeled dendrite. G, Surface reconstruction of the dendrite shown in F color coded for synaptotagmin-staining intensity. $H$, Additional high-resolution single optical section of labeled dendrites (red) and synaptotagmin (green). Arrows indicate synaptotagmin spots associated with labeled dendrites.Asterisks indicate synaptotagmin spots not in contact with the labeled dendrites. Scale bars: $A, B, D, 10 \mu \mathrm{m} ; C$, $1 \mu \mathrm{m} ; E, 5 \mu \mathrm{m} ; F-H, 0.3 \mu \mathrm{m}$.

each of these sites represented an input synapse, but rather that a close spatial association between the dendritic surface and synaptic proteins indicated a high likelihood for a synaptic connection, which we visualized as a color code on the surface reconstruction.
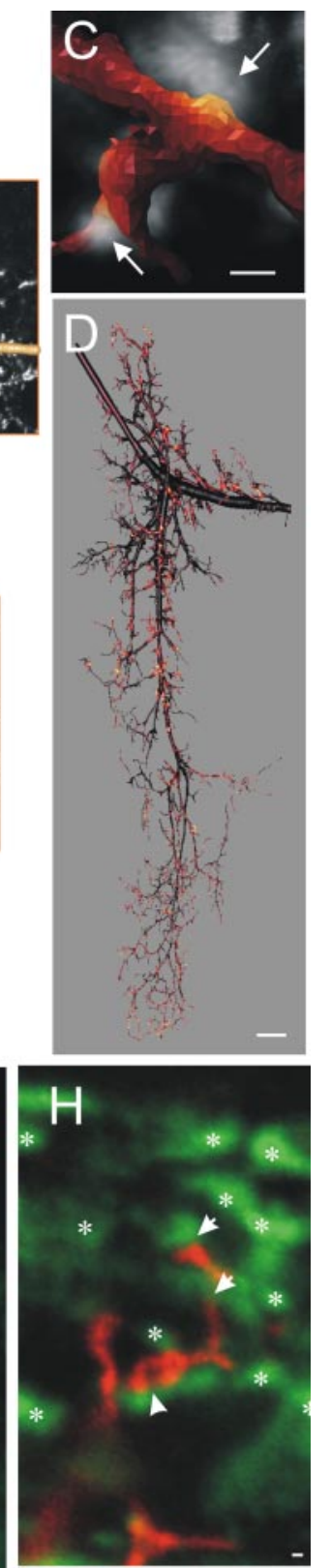

This procedure was then applied for entire dendritic trees (Fig. 5D).

When applying this analysis to the dendritic trees from the stimulated and contralateral unstimulated MN5 from the same ganglion, many more putative synaptic input sites were marked on the surface reconstruction of the stimulated motoneuron (Fig. 6A). For better visualization of the color code, selective enlargements of comparable dendritic areas from the stimulated and the control dendritic tree are depicted in Figures $6 D-G$. White arrowheads point at sites with high synaptotagmin staining intensities within a $0.3 \mu \mathrm{m}$ vicinity of the dendritic surface (Fig. 6D,F). Throughout all parts of the dendritic field and dendrites of almost all orders, many more putative sites of input synapses were detected at the dendritic tree of the stimulated compared with the control motoneuron (Fig. 6A, $D-G$ ). Each threedimensional reconstruction of the dendritic field consisted of reconstructed compartments with $0.5 \mu \mathrm{m}$ length (see Materials and Methods). To address the distribution of putative synaptic input sites throughout the dendritic tree, all reconstructed compartments with high synaptotagmin staining intensities within a $0.3 \mu \mathrm{m}$ vicinity of their surface were counted in stimulated and in contralateral, nonstimulated motoneurons from the same preparations. Plotting the number of compartments with high synaptotagmin staining intensities in their close vicinity as a function of branch order for a representative animal showed that in the stimulated dendritic tree in all branch orders above 10 more putative sites of input synapses occurred compared with the control dendritic tree (Fig. 6B). In contrast, stimulation led to an increased number of dendritic branches only in the higher branch orders (between branch order 30 and 60) (Fig. 6C). Therefore, dendritic regression was decreased mostly in fine highorder branches (Figs. 2, 6F), whereas elimination of putative input synapses was decreased throughout almost the entire dendritic field. Comparing the number of reconstructed compartments with high synaptotagmin staining intensity within a $0.3 \mu \mathrm{m}$ vicinity of their surface in three pairs of control and stimulated dendritic trees, each pair derived from a single preparation (stimulated MN5 and nonstimulated internal contralateral control MN5) revealed that stimulated motoneurons received significantly more putative input synapses compared with nonstimulated internal controls (Fig. 6I) (paired Student's $t$ test; $p<0.01$ ). 
During normal development, the number of putative input synapses decreased significantly between the stages W2 and P0 (data not shown), corresponding to the physiological findings in this study and earlier findings on synapse elimination in Manduca during late larval life (Streichert and Weeks, 1995; Gray and Weeks, 2003). The anatomical observation of more putative input synapses on the surface of the dendrites of the stimulated MN5 compared with the internal control corresponded to physiological measurements from the same neurons. Cumulative probability plots of individual postsynaptic potentials showed that there was an increase in larger amplitude potentials in the stimulated compared with the control MN5 (Fig. 6H).

\section{Discussion}

During the metamorphosis of amphibians (Alley and Omerza, 1998) and holometabolous insects (Consoulas et al., 2000; Tissot and Stocker, 2000), reutilization of motoneurons for new adult behaviors is characterized by the retraction of axonal processes from degenerating larval muscles followed by outgrowth on the newly formed adult muscles and by retraction of larval dendrites followed by the maturation of the adult dendritic architecture. In holometabolous insects, dendritic regression is dependent on hormonal cues (Weeks et al., 1992; Weeks, 1999; Williams and Truman, 2004). Manduca proleg motoneurons are one of the rare systems in which it has been shown that dendritic retraction is accompanied by synapse elimination and, thus, the dismantling of larval motor circuits (Streichert and Weeks, 1995; Gray and Weeks, 2003). The present study supports this view by showing that regression of MN5 dendrites went along with a marked reduction in the number of putative input synapses, most likely resembling the dismantling of the larval crawling circuits.

During normal development, larval circuit dismantling is accompanied by a phase of reduced movement after the animals burrowed underground awaiting pupation (Reinecke et al., 1980; Dominick and Truman, 1994a). During this phase, the spiking activity of MN5 ceases significantly (Duch and Mentel, 2003). We used chronically implanted extracellular electrodes to test whether changes in the normal decrease in spiking activity in MN5 would interfere with dendritic regression and larval circuit dismantling. This approach had two important advantages. First, we were able to manipulate the activ-
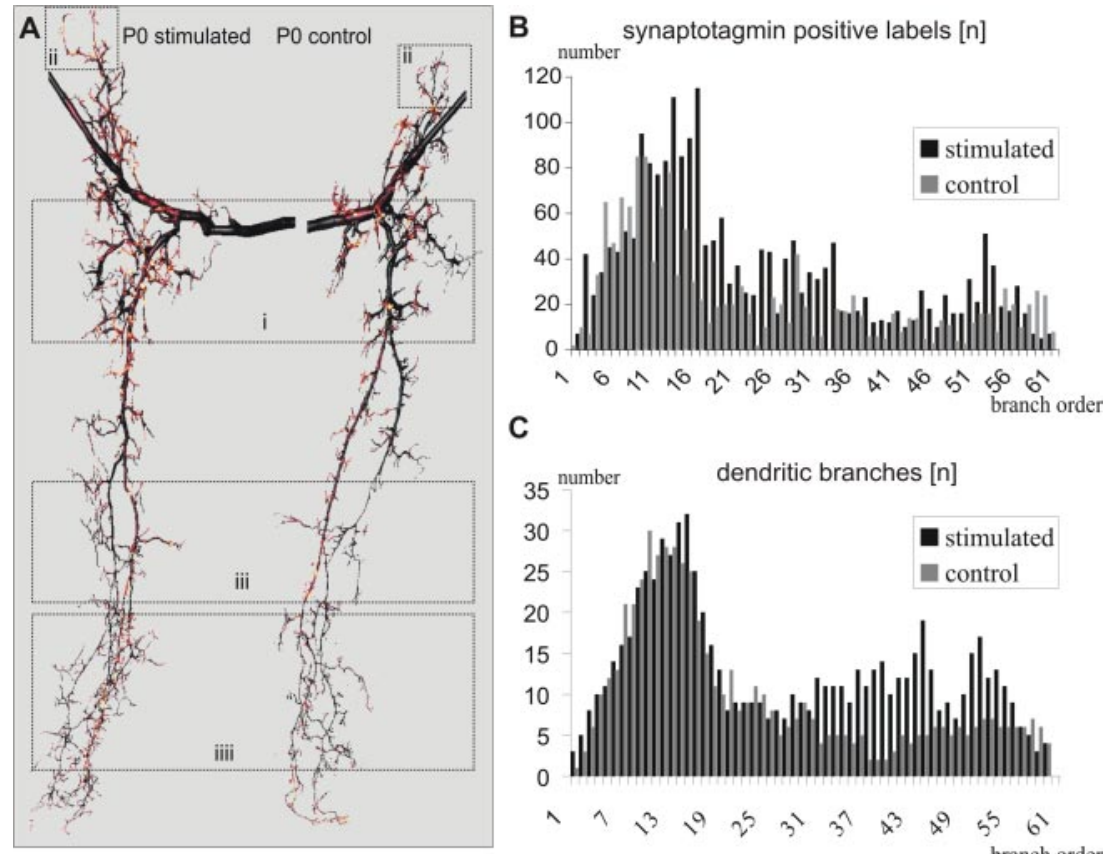

C
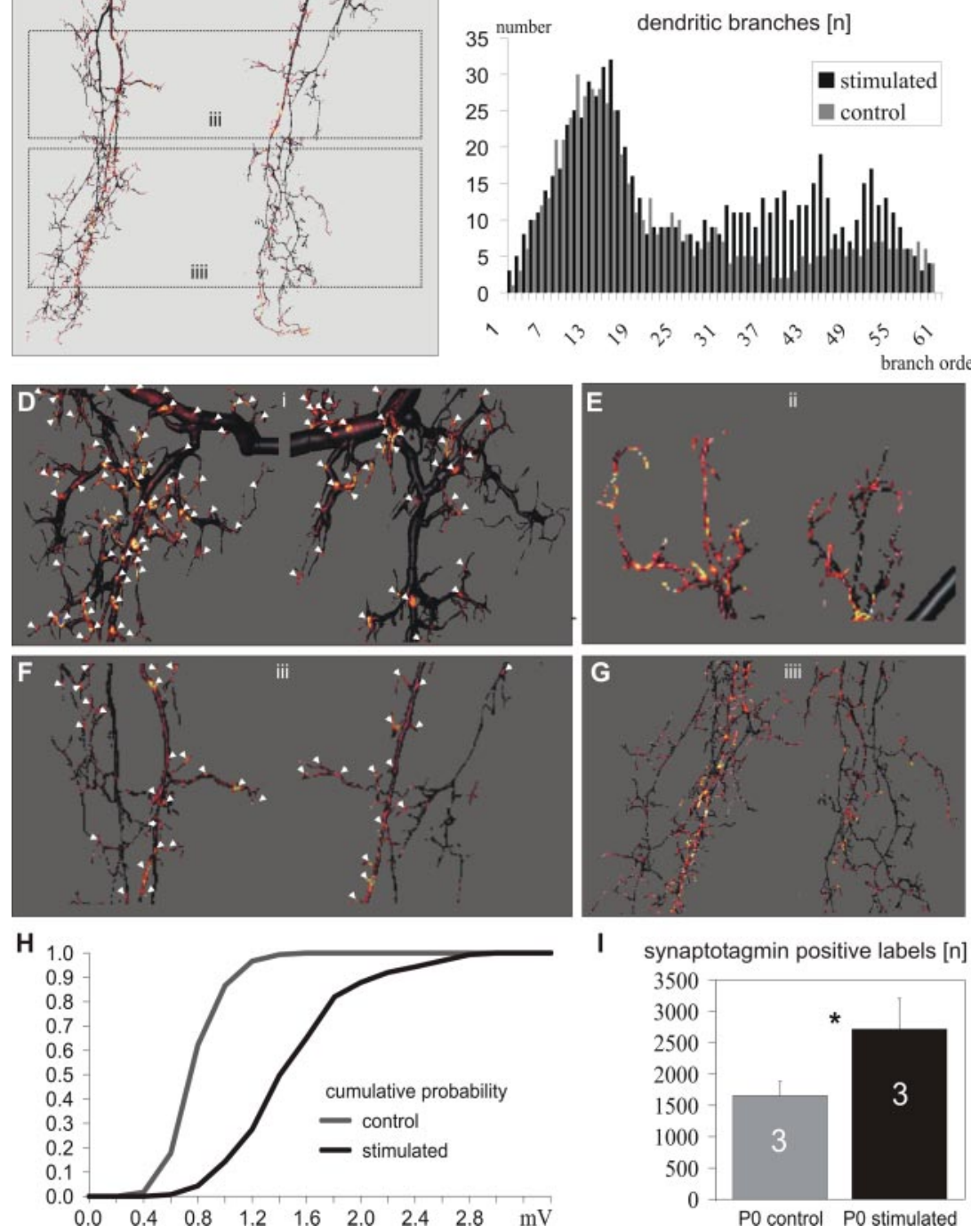

I synaptotagmin positive labels [n]

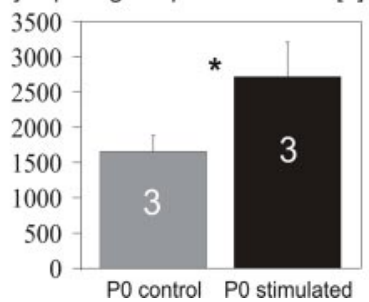

Figure 6. Effects of stimulation on distribution of putative input synapses. $A$, The intensity of synaptotagmin immunostaining within $0.3 \mu \mathrm{m}$ distance from the dendritic surface is shown as a color code throughout the entire dendritic tree of the stimulated (left) and the control (right) MN5 from the same ganglion. Warmer colors represent a higher probability for putative input synapses. The stimulated dendritic tree (left) shows more spots with warmer colors compared with the control tree (right). Selective enlargements of the parts of the dendritic trees surrounded by the boxes $i$-iiii are shown in $D-G$. In $D$ and $F$, spots with a high probability for putative input synapses are marked by arrowheads. The stimulated dendritic tree showed more sites of high-input synapse probability than the control tree on both high-order dendrites, which are not present only in the stimulated tree, and low-order dendrites, which are present in both trees. B, All three-dimensional reconstructions were composed of multiple compartments of $0.5 \mu$ m length each. The number of compartments with a high probability for putative input synapses plotted as a function of branch order for the stimulated versus the control MN5. The stimulated dendritic tree received more putative input synapses in all branch orders larger than 10. C, The number of dendritic branches as a function of branch order for the stimulated versus the control MN5. The stimulated MN5 contains more branches in the orders $30-60 . \mathrm{H}$, Cumulative probability plots of individual postsynaptic potentials for the control versus the stimulated MN5. The increase in larger amplitude potentials in the stimulated compared with the control MN5 corresponded to the higher number of putative input synapses detected anatomically $(B, D-G) . I$, The average number of reconstructed compartments with a high probability for putative input synapses counted in three pairs of control and stimulated dendritic trees, each pair derived from a single preparation. Error bars represent SDs. Asterisk indicates significance (paired Student's t test; $p<0.01$ ). 
ity of a subset of identified motoneurons during selected periods of development only, whereas all other neurons were unaffected and the animals developed normally. In genetic model systems, this is only possible by the expression of mutant alleles that cause hyperexcitability or hypoexcitability in subsets of neurons and can be controlled in a temporal manner (Rubin, 1988). Second, we could manipulate spiking activity on the basis of our knowledge of the alterations in activity during normal development (Duch and Mentel, 2003). The results clearly showed that stimulating MN5 during a developmental time when it usually showed only few spikes did not stop but significantly decreased dendritic regression and synapse elimination. This agreed with steroid control of dendritic regression (Weeks et al., 1992; Knittel and Kent, 2002), but it also clearly showed that activity significantly decreased both dendritic regression and synapse elimination. The possibility that motoneuron activity might, in turn, be controlled by steroid hormones or downstream peptide cascades is discussed previously (Duch and Mentel, 2003). However, it appeared unlikely that steroid control of dendritic regression might entirely be mediated via changes in activity, because stimulation experiments did not stop but reduced dendritic regression, even at higher stimulation frequencies (data not shown).

Dendritic regression and the effects of induced spiking activity were quantified by geometric reconstructions of the dendritic field of MN5. The effects of activity on synapse elimination were analyzed physiologically and anatomically. The results demonstrated that amplitude and frequency of spontaneously occurring PSPs, as recorded from the soma of MN5, were significantly increased in stimulated neurons. Because neither physiological parameters like spike shape and input resistance nor geometric parameters like dendritic diameters were affected by the stimulation, increased PSP amplitude most likely resembled increased synaptic input. This was further supported by anatomical results showing more immunocytochemically labeled synaptotagmin profiles in close proximity to the dendritic surface of the stimulated compared with the control MN5. Electron microscopy in other insects demonstrated that the distribution of synapsin I-protein and synaptotagmin-protein is useful in detecting synaptic loci at the light microscopic level (Watson and Schurmann, 2003). Paired with the precise surface definitions of our geometric neuron reconstructions, synaptotagmin profiles can be used to estimate the distribution of putative input synapses throughout the entire dendritic tree of MN5 on the light microscopy level (Schmitt et al., 2004). We do not claim that every synaptotagmin label within $0.3 \mu \mathrm{m}$ of the dendritic surface resembles an input synapse, but rather that a close spatial association between the dendritic surface and synaptic proteins indicates a high likelihood for a synaptic connection. Corresponding to the physiological results, this "synaptic contact probability" was strongly increased in stimulated versus control motoneurons. This suggested that activity decreased steroid-induced synapse elimination in the CNS.

In vivo stimulation of nerve 1 led to antidromic action potentials in MN5 and few other efferent neurons (see Materials and Methods) (Duch and Mentel, 2003). Because most efferent insect neurons contain no, or only very few, central output synapses (Burrows et al., 1989; Watson and Schurmann, 2003) decreased dendritic regression in MN5 was most likely caused by intrinsic activity and not by synaptic activity, although we cannot fully exclude the possibility of increased synaptic activity in the motor networks resulting from the stimulation experiments. The mechanism by which increased activity mediated decreased dendritic regression remains to be investigated. Although at current spec- ulative, it seems logical that common cell biological mechanisms might be co-opted by hormonal signals and activity-dependent programs (Eaton and Davis, 2003). Antidromically induced spiking activity in the developing MN5 induced calcium elevations in the soma and possibly also in large parts of the dendrites, depending on developmental changes in the ion channel distribution (Duch and Levine, 2002). Alterations in somatic calcium concentration can affect gene transcription (Hardingham et al., 1997; West et al., 2002), which in turn can affect dendritic shape. In cortical slice culture, dendritic shape is dependent on CaM kinase IV activity and the cAMP-responsive element-binding protein (Redmond et al., 2002). Therefore, activity and steroid hormone action might converge on the level of gene transcription.

The observation that dendritic regression was reduced, but no dendritic growth was induced, by activity contrasted with earlier results on stimulation-induced axonal outgrowth of MN5 (Duch and Mentel, 2003). This is not surprising because axons and dendrites clearly differ in their patterns of growth and in their molecular composition (Ramakers et al., 2001; Nikonenko et al., 2003; Tashiro et al., 2003). Similarly, hormonal manipulation studies have shown that axonal and dendritic regression occurred independently from each other in heterochronic mosaic animals (Knittel and Kent, 2002), suggesting a different set of signals for regressive events in the periphery nervous system and the CNS. These findings also concur with the events occurring during Wallerian degeneration in the mouse, where synaptic degeneration may be mediated by different mechanisms than axon and soma degeneration (Gillingwater and Ribchester, 2001).

The elimination of previously functional synaptic connections has been demonstrated in the vertebrate (Purves and Lichtman, 1980; Nguyen and Lichtman, 1996; Colman et al., 1997; Sanes and Lichtman, 1999; Chen and Regehr, 2000) and in the invertebrate CNS and peripheral nervous system (Sonea and Rheuben, 1992; Streichert and Weeks, 1995; Truman and Reiss, 1995; Consoulas and Levine, 1998; Duch et al., 2000; Eaton et al., 2002; Gray and Weeks, 2003). However, the mechanisms that regulate circuit dismantling are not well understood (Goda and Davis, 2003). Many studies in the CNS and the periphery nervous system support the conclusion that synapse elimination is mediated by activity-dependent competition mediated through the postsynaptic cell (Katz and Shatz, 1996; Sanes and Lichtman, 1999). The postsynaptic cell as intermediary is further supported by differential synapse elimination of a single motor neuron at the vertebrate neuromuscular junction, arguing for local control at each muscle fiber (Keller-Peck et al., 2001). For Manduca proleg motoneurons, it has been suggested recently that steroidinduced dendritic regression physically disconnected dendrites from the synaptic terminals of sensory neurons, producing synaptic weakening (Gray and Weeks, 2003). In contrast, this study suggests at least in part a differential effect of induced activity on dendrites versus synapses, because synapse elimination was decreased in all branch orders above 10, whereas dendritic regression was decreased only in high branch orders above 30 . Although the mechanisms for either process remain speculative at present, the results strengthen the role of the postsynaptic cell for synapse elimination because alterations were induced by stimulating the postsynaptic neuron.

In wandering Manduca larvae, activity-dependent modulation of dendritic regression and synaptic weakening is most likely of behavioral importance. Despite the ongoing degeneration of the larval target muscle of MN5, neuromuscular transmission and muscle contraction remain functional until pupal life (Duch et al., 2000), and MN5 participates in crawling, burrowing, and 
ecdysis behavior (Duch and Mentel, 2003). In case of delayed wandering, motoneuron activity will remain higher compared with normal development. This increased activity will then most likely delay dendritic regression and synapse elimination so that the adequate performance of larval behavior will not be impaired too early by an inflexible hormone program. Activity-dependent modulation of steroid-induced developmental programs was also shown for programmed cell death (PCD). Motoneuron PCD could be delayed by ligating connectives in Manduca (Fahrbach and Truman, 1987) or by forcing emerging flies to show prolonged ecdysis behavior (Kimura and Truman, 1990).

In conclusion, our findings represent the first demonstration of activity-dependent modulation of steroid-induced dendritic regression and synapse elimination. Chronic extracellular recordings and stimulation make holometabolous insects suitable to further elucidate the concerted interplay between hormonal signals and activity-dependent mechanisms for structural and physiological changes of motoneurons.

\section{References}

Alley KE, Omerza FF (1998) Reutilization of trigeminal motoneurons and metamorphic reorganization of the jaw myoneural systems in frogs. Brain Res 813:187-190.

Bell RA, Joachim FA (1976) Techniques for rearing laboratory colonies of tobacco hornworms and pink ballworms. Ann Entomol Soc Am 69:365-373.

Bollenbacher WE, Smith SL, Goodman W, Gilbert LI (1981) Ecdysteroid titer during the larval-pupal-adult development of the tobacco hornworm, Manduca sexta. Gen Comp Endocrinol 44:302-306.

Bottjer SW, Arnold AP (1997) Developmental plasticity in neural circuits for a learned behavior. Annu Rev Neurosci 20:459-481.

Burrows M, Watson AHD, Brunn DE (1989) Physiological and ultrastructural characterisation of a central synaptic connection between identified motoneurons in the locust. Eur J Neurosci 1:111-126.

Caselles V, Kimmer R, Sapiro G (1997) Geodesic active contours. Int J Comp Vision 22:61-79.

Chen C, Regehr WG (2000) Developmental remodeling of the retinogeniculate synapse. Neuron 28:955-966.

Cline HT (2001) Dendritic arbor development and synaptogenesis. Curr Opin Neurobiol 11:118-126.

Colman H, Nebekura J, Lichtman JW (1997) Alterations in synaptic strength preceding axon withdrawal. Science 275:356-361.

Consoulas C, Levine RB (1998) Presynaptic function during muscle remodeling in insect metamorphosis. J Neurosci 18:5817-5831.

Consoulas C, Kent KS, Levine RB (1996) Remodeling of the peripheral processes and presynaptic terminals of leg motoneurons during metamorphosis of the hawkmoth, Manduca sexta. J Comp Neurol 372:415-434.

Consoulas C, Duch C, Bayline RJ, Levine RB (2000) Behavioral transformations during metamorphosis: remodeling of neural and motor systems. Brain Res Bull 53:571-583.

Dominick OS, Truman JW (1984a) The physiology of wandering behavior in Manduca sexta. I. Temporal organization and the influence of the internal and external environments. J Exp Biol 110:35-51.

Dominick OS, Truman JW (1984b) The physiology of wandering behavior in Manduca sexta. II. The endocrine control of wandering behavior. J Exp Biol 110:35-51.

Dubuque SH, Schachtner J, Nighorn AJ, Menon KP, Zinn K, Tolbert LP (2001) Immunolocalization of synaptotagmin for the study of synapses in the developing antennal lobe of Manduca sexta. J Comp Neurol 444:277-287.

Duch C, Levine RB (2000) Remodeling of membrane properties and dendritic architecture accompany the postembryonic conversion of a slow into a fast motoneurons. J Neurosci 20:6950-6961.

Duch C, Levine RB (2002) Changes in calcium signaling during postembryonic dendritic growth in Manduca sexta. J Neurophysiol 87:1415-1425.

Duch C, Mentel T (2003) Stage-specific activity patterns affect motoneuron axonal retraction and outgrowth during the metamorphosis of Manduca sexta. Eur J Neurosci 17:945-962.

Duch C, Bayline RJ, Levine RB (2000) Postembryonic development of the dorsal longitudinal flight muscle and its innervation in Manduca sexta. J Comp Neurol 422:1-17.

Eaton BA, Davis GW (2003) Synaptic disassembly. Genes Dev 17:2075-2082.

Eaton BA, Fetter RD, Davis GW (2002) Dynactin is necessary for synapse stabilization. Neuron 3:729-741.

Fahrbach SE, Truman JW (1987) Possible interactions of a steroid hormone and neural inputs in controlling the death of an identified neuron in the moth Manduca sexta. J Neurobiol 18:497-508.

Fahrbach SE, Weeks JC (2002) Hormonal regulation of neural and behavioral plasticity in insects. In: Hormones, brain, and behavior, Vol 3 (Pfaff D, Arnold A, Etgen A, Fahrbach S, Rubin R, eds), pp 331-358. San Diego: Academic.

Gillingwater TH, Ribchester RR (2001) Compartmental neurodegeneration and synaptic plasticity in the Wld(s) mutant mouse. J Physiol (Lond) 543:627-639.

Goda Y, Davis GW (2003) Mechanisms of synapse assembly and disassembly. Neuron 40:243-264.

Gray JR, Weeks JC (2003) Steroid-induced dendritic regression reduces anatomical contacts between neurons during synaptic weakening and the developmental loss of a behavior. J Neurosci 23:1406-1415.

Hardingham GE, Chawla S, Johnon CM, Bading H (1997) Distinct functions of nuclear and cytoplasmatic calcium in the control of gene expression. Nature 385:260-265.

Kass M, Wittkin A, Terzopoulos D (1988) Snakes: active contour models. Int J Comp Vision 1:321-331.

Katz LC, Shatz CJ (1996) Synaptic activity and the construction of cortical circuits. Science 274:1133-1138.

Keller-Peck CR, Walsh MK, Gan WB, Feng G, Sanes JR, Lichtman JW (2001) Asynchronous synapse elimination in neonatal motor units: studies using GFP transgenic mice. Neuron 31:381-394.

Kimura HI, Truman JW (1990) Postmetamorphic cell death in the nervous and muscular systems of Drosophila melanogaster. J Neurosci 10:403-411.

Knittel LM, Kent KS (2002) Remodeling of an identified motoneuron during metamorphosis: central and peripheral actions of ecdysteroids during regression of dendrites and motor terminals. J Neurobiol 52:99-116.

Levine RB, Weeks JC (1989) Reorganization of neural circuits and behavior during insect metamorphosis. In: Perspectives in neural circuits and behavior (Carew TJ, Kelley D, eds), pp 195-228. New York: Liss.

Levine RB, Weeks JC (1996) Cell culture approaches to understanding the actions of steroid hormones on the insect nervous system. Dev Neurosci 18:73-86.

Levine RB, Morton DB, Restifo LL (1995) Remodeling of the insect nervous system. Curr Opin Neurobiol 5:28-35.

Libersat F, Duch C (2002) Morphometric analysis of dendritic remodeling in an identified motoneuron during postembryonic development. J Comp Neurol 450:153-166.

Libersat F, Duch C (2004) Mechanisms of dendritic maturation. Mol Neurobiol 3:303-320.

McAllister AK (2000) Cellular and molecular mechanisms of dendrite growth. Cereb Cortex 10:963-973.

Miles CI, Weeks JC (1991) Developmental attenuation of pre-ecdysis motor pattern in the tobacco hornworm, Manduca sexta. J Comp Physiol [A] 168:179-190.

Nguyen QT, Lichtman JW (1996) Mechanism of synapse disassembly at the developing neuromuscular junction. Curr Opin Neurobiol 6:104-112.

Nijhout HF, Williams CM (1974) Control of molting and metamorphosis in the tobacco hornworm, Manduca sexta (L): growth of the last instar larva and the decision to pupate. J Exp Biol 61:481-491.

Nikonenko I, Jourdain P, Muller D (2003) Presynaptic remodeling contributes to activity-dependent synaptogenesis. J Neurosci 23:8498-84505.

Peters BH, Altman JS, Tyrer NM (1985) Synaptic connections between the hindwing stretch receptor and flight motor neurones in the locust revealed by double cobalt labelling for electron microscopy. J Comp Neurol 233:269-284.

Purves D, Lichtman JW (1980) Elimination of synapses in the developing nervous system. Science 210:153-157.

Ramakers GJ, Avci B, van Hulten P, van Ooyen A, van Pelt J, Pool CW, Lequin MB (2001) The role of calcium signaling in early axonal and dendritic morphogenesis of rat cerebral cortex neurons under non-stimulated growth conditions. Brain Res Dev Brain Res 126:163-172.

Redmond L, Kashani A, Gosh A (2002) Calcium regulation of dendritic 
growth via CaM kinase IV and Creb-mediated transcription. Neuron 34:999-1010.

Reinecke JP, Buckner JS, Grugel SR (1980) Life cycle of laboratory reared tobacco hornworms, Manduca sexta, a study of development and behavior, using time lapse cinematography. Biol Bull Mar Lab Woods Hole 158:129-140.

Riddiford LM, Truman JW (1994) Hormone receptors and the orchestration of development during insect metamorphosis. In: Perspectives in comparative endocrinology, pp 389-394. Ottawa, Canada: National Research Council of Canada.

Rubin GM (1988) Drosophila melanogaster as an experimental organism. Science 240:1453-1459.

Sanes JR, Lichtman JW (1999) Development of the vertebrate neuromuscular junction. Annu Rev Neurosci 22:389-442.

Schmitt S, Evers JF, Scholz M, Duch C, Obermayer K (2004) New methods for the computer assisted 3D reconstruction of neurons from confocal image stacks. NeuroImage, in press.

Sonea IM, Rheuben MB (1992) Degenerative changes in the function of neuromuscular junctions of Manduca sexta during metamorphosis. J Exp Biol 167:61-89.

Streichert LC, Weeks JC (1995) Decreased monosynaptic sensory input to an identified motoneuron is associated with steroid-mediated dendritic regression during metamorphosis in Manduca sexta. J Neurosci 15:1484-1495.

Tashiro A, Dunaevsky A, Blazeski R, Mason CA, Yuste R (2003) Bidirectional regulation of hippocampal mossy fiber filopodial motility by kainate receptors: a two-step model of synaptogenesis. Neuron 38:773-784.

Tissot M, Stocker RF (2000) Metamorphosis of Drosophila and other insects: the fate of neurons throughout stages. Prog Neurobiol 62:89-111.

Toran-Allerand CD, Singh M, Setalo Jr G (1999) Novel mechanisms of estrogen action in the brain: new players in an old story. Front Neuroendocrinol 2:97-121.

Truman JW (1990) Metamorphosis of the insect nervous system. In: Metamorphosis: postembryonic reprogramming of gene expression in amphibian and insect cells (Gilbert LI, Tata JR, Atkinson BG, eds), pp 283320. San Diego: Academic.

Truman JW, Reiss SE (1995) Neuromuscular metamorphosis in the moth Manduca sexta: hormonal regulation of synapses loss and remodeling. J Neurosci 15:4815-4826.
Truman JW, Taghert PH, Reynolds SE (1980) Physiology of pupal ecdysisin the tobacco hornworm, Manduca sexta. J Exp Biol 88:327-337.

Watson AH, Schurmann FW (2002) Synaptic structure, distribution, and circuitry in the central nervous system of the locust and related insects. Microscopy Res Tech 56:210-226.

Weeks JC (1999) Steroid hormones, dendritic remodeling and neuronal death: insights from insect metamorphosis. Brain Behav Evol 54:51-60.

Weeks JC, Truman JW (1985) Independent steroid control of the fates of motoneurons and their muscles during insect metamorphosis. J Neurosci 5:2290-2300.

Weeks JC, Truman JW (1986) Hormonally mediated reprogramming of muscles and motoneurones during the larval-pupal transformation of the tobacco hornworm, Manduca sexta. J Exp Biol 125:1-13.

Weeks JC, Roberts WM, Trimble DL (1992) Hormonal regulation and segmental specificity of motoneuron phenotype during metamorphosis in Manduca sexta. Dev Biol 149:185-196.

West AE, Griffith EC, Greenberg ME (2002) Regulation of transcription factors by neuronal activity. Nat Rev Neurosci 3:921-931.

Williams DW, Truman JW (2004) Mechanisms of dendritic elaboration of sensory neurons in Drosophila: insights from in vivo time lapse. J Neurosci 24:1541-1550.

Wong ROL, Gosh A (2002) Activity-dependent regulation of dendritic growth and patterning. Nat Rev Neurosci 3:803-812.

Woolley CS (1998) Estrogen-mediated structural and functional synaptic plasticity in the female rat hippocampus. Horm Behav 34:140-148.

Woolley CS, McEwen BS (1994) Estradiol regulates hippocampal dendritic spine density via an N-methyl-D-aspartate. J Neurosci 14:7680-7687.

Woolley CS, Weiland NG, McEwen BS, Schwartzkroin PA (1997) Estradiol increases the sensitivity of hippocampal CA1 pyramidal cells to NMDA receptor-mediated synaptic input: correlation with dendritic spine density. J Neurosci 17:1848-1859.

Wouterlood FG, Van Denderen JC, Blijleven N, Van Minnen J, Hartig W (1998) Two-laser dual-immunofluorescence confocal laser scanning microscopy using Cy2- and Cy5-conjugated secondary antibodies: unequivocal detection of co-localization of neuronal markers. Brain Res Brain Res Protoc 2:149-159.

Zar JH (1984) Biostatistical analysis, Ed 2. Englewood Cliffs, NJ: Prentice Hall. 